\title{
Realization of THz Band Mixer Using Graphene
}

\author{
Ensieh Ghasemi Mizuji ${ }^{1}$, Ali Abdolali ${ }^{1}$, Fateme Aghamohamadi $^{1}$, \\ Mohammad Danaeifar ${ }^{2}$, Soheil Hashemi ${ }^{1}$, and Kimia Nikooei Tehrani ${ }^{1}$ \\ ${ }^{1}$ Department of Electrical Engineering, Iran university of science and technology, Tehran, Iran \\ ${ }^{1}$ Department of Electrical Engineering, K.N.Toosi university of technology, Tehran, Iran \\ *corresponding author, E-mail: ensiyeh.ghasemi@ee.iust.ac.ir
}

\begin{abstract}
In this article a new method for creating mixer component in infrared and $\mathrm{THz}$ is suggested. Since the nonlinear property of admittance creates frequency components that do not exist in the input signal and the electrical conductivity is associated with admittance, in our work we have proven and simulated that the nonlinear property of graphene admittance can produce mixer component. The simulation results show that the mixer component is larger than other components, therefore the mixer works properly. Because of nano scale of graphene structure, this method paves the road to achieve super compact circuits.
\end{abstract}

\section{Introduction}

Mixers are used to shift signals from one frequency range to another range of frequencies. For example mixer converts the intermediate signals produced by a generator to RF signals, This procedure is named Up Conversion. The same operation is performed in receiver.

Graphene is the first example of two dimensional structures with one atom thickness that have received much attention in recent years[1,10]. Its unique electrical and photonic properties have several usages in the optical and $\mathrm{THz}$ circuits and systems. One basic parameter in describing graphene optical properties is its complex conductivity, $\sigma_{\mathrm{g}}=$ $\sigma_{\mathrm{gr}} \pm \mathrm{i} \sigma_{\mathrm{gi}}$ which depends on frequency $\omega$ of incident wave, $\Gamma$ charged particle scattering rate which represents loss mechanism, temperature $\mathrm{T}$, and chemical potential $\mu_{\mathrm{c}}$ which depends on the carrier density and can be controlled by a gate voltage that produces electric field, magnetic field and/or chemical doping [11,12], hence conductivity is frequency dependent and controllable by varying its chemical potential. Due to the complex conductivity, equivalent circuit including a resistor and a capacitor or an inductor may be considered. Graphene is suitable for realizing transistor actions due to High intrinsic carrier mobility and speed[13,14,15]. Recently, graphene has been utilized for devising subharmonic resistive mixers[16]. In this article we demonstrate that the nonlinear conductance property can make biased graphene suitable for mixer application.

There are other technologies such as schottky barrier diodes and Hot-Electron bolometer for mixers. A small area barrier between a metal and a semiconductor can be used as
Schottky barrier diode. A strongly nonlinear current-voltage characteristic makes Schottky barrier diode useful for mixing application. A major disadvantage is that relatively high level of LO power is required for SBD (Schottky Barrier Diode) to act as a nonlinear device for optimum performance as a mixer. Furthermore, SBD is more sensitive than other types of devices which have stronger nonlinearities. The other method is Hot-Electron bolometer. In this method, the bolometer output signal is proportional to the power of the incident radiation. To utilize it as heterodyne detector the bolometer should be fast enough to follow the fast IF amplitude modulation of a RF signal. The major disadvantage of this type of mixer is poor electrical stability. Our mixer is $2 \mathrm{D}$ and can reduce the scale of the circuits to nano. Also due to tunable nature of graphene this scheme would be broadband. Its strong nonlinearity has given graphene good performance as a mixer. Simulation results confirm our conclusion. Section II describes the electronic model of graphene. Section III is a brief explanation of the action of nonlinear conductivity in mixer application. Section IV surveys graphene as a nonlinear conductivity and this characterization is proved analytically and in simulation.

\section{Electronic model of graphene}

Graphene is the 2D kind of graphite and modeled as one atom thickness material sheet with a surface conductivity tensor[17]. Graphene electrical conductivity is considered as[17]:

$\sigma\left(\omega, \mu_{c}\left(E_{0}\right), \Gamma, T, B_{0}\right)=x x \sigma_{x x}+x y \sigma_{x y}+y x \sigma_{y x}+y^{\wedge} y^{\wedge} \sigma_{y y}$

Where $\omega$ is the radian frequency, $\mu_{\mathrm{c}}$ is the chemical potential (which may vary by the electric and/or magnetic fields), $\mathrm{E}_{0}=\mathrm{E}_{0}$ and $\mathrm{B}_{0} \quad{ }_{0}$ are the induced electric and magnetic fields, respectively, $\Gamma$ is the electron scattering rate and $\mathrm{T}$ is temperature. Three general cases for graphene conductivity are considered. We assume the graphene conductivity in three types of bias such as: without any electric bias or magnetic bias with spatial dispersion, with an electric bias and with a magnetic bias. Here we consider second type for graphene. So the formulation for the electrical conductivity of graphene will be[11]:

$\sigma_{x x}=\sigma_{y y}=\sigma_{d}\left(\mu_{c}\left(E_{0}\right)\right)$

$\sigma_{x y}=\sigma_{y x}=0$ 
Using the above relations and kubo formula the electrical conductivity is[11]:

$$
\begin{aligned}
& \sigma_{g}\left(\omega, \mu_{c}, \Gamma, T\right)=\frac{-i e^{2}(\omega+i 2 \Gamma)}{\pi h^{2}}\left[\frac { 1 } { ( \omega + i 2 \Gamma ) ^ { 2 } } \int _ { 0 } ^ { \infty } \Omega \left(\frac{\partial f_{d}(\Omega)}{\partial \Omega}-\frac{\partial f_{d}(-\Omega)}{\partial \Omega} d \Omega\right.\right. \\
& \left.-\int_{0}^{\infty} \frac{f_{d}(-\Omega)-f_{d}(\Omega)}{(\omega+i 2 \Gamma)^{2}-4(\Omega / \hbar)^{2}} d \Omega\right]
\end{aligned}
$$

Where $f_{d}(\Omega)=\left[e^{\left(\Omega-\mu_{c} / k_{B} T\right]}+1\right]^{-1}$.

If $\sigma_{\mathrm{g}}$ is considered as $\sigma_{\mathrm{g}} \sigma_{\text {interband }}+\sigma_{\text {intraband, }}$, real and imaginary part may be estimated as below:

$$
\begin{aligned}
\sigma_{\text {interband }} & =\frac{i e^{2}}{4 \pi \hbar} \ln \left(\frac{2\left|\mu_{c}\right|-(\omega+i 2 \Gamma) \hbar}{2\left|\mu_{c}\right|+(\omega+i 2 \Gamma) \hbar}\right) \\
\sigma_{\text {intraband }} & =\frac{i e^{2} k_{B} T}{\pi \hbar^{2}(\omega+i 2 \Gamma)}\left(\frac{\mu_{c}}{k_{B} T}+\ln \left(e^{-\frac{\mu_{c}}{k_{B} T}}+1\right)\right)
\end{aligned}
$$

Figure 1 shows the real and imaginary parts of the graphene conductivity.

The equivalent circuit of graphene is considered as a resistor that represents loss and a parallel capacitor or inductor Due to imaginary part of conductivity being positive or negative (figure 2).

\section{Application of the non-linear conductivity of graphene for realizing mixing action}

Graphene has nonlinear characterization that relates its voltage to its current. Since nonlinearity produces output frequencies that do not exist in input signal, we may use the nonlinear conduction property for mixer application as follows [18]:

$I=I(v)$

This system is biased by $V(t)=V_{L} \cos \left(\omega_{L} t\right)+v(t)$ in which $V_{L} \cos \left(\omega_{L} t\right)$ is a large signal and $v(t)$ is a small signal where $\mathrm{V}_{\mathrm{L}}$ is the amplitude of the large signal. The large signal acts as a time variant bias. We may ignore high order terms due to the small voltage, so that we assume that the system acts linearly for small signal. By expanding the current around the large signal, $V_{L} \cos \left(\omega_{L} t\right)$, equation 7 is obtained.

$$
\begin{aligned}
& I=f\left(v_{L}(t)\right)+\left.\frac{\partial f}{\partial v}\right|_{v_{L}(t)} v(t)+\left.\frac{\partial^{2} f}{\partial v^{2}}\right|_{v_{L}(t)} v^{2}(t)+\ldots \\
& i=I-f\left(v_{L}(t)\right)=g_{1}(t) v(t)+g_{2}(t) v^{2}(t)+\ldots
\end{aligned}
$$

The terms with odd exponent produce terms like $b_{n} \cos \left(\omega_{L} t\right) \cos \left(\omega_{s} t\right)$, so we can use the first component as a mixer term and the third as an inter-modulation term. Since higher order terms have smaller values, the saturation occurs later.

\section{Non-linear conduction of the graphene and the role of mixing}

As shown in Figure 3, the conductivity of the graphene, associated with its admittance, is non-linear versus the chemical voltage. Therefore, we can use it as a mixer. From [18] we know that when $\mu_{c}$ is varying in small range, the relation between $\mathrm{E}$ and $\mu_{\mathrm{c}}$ can be approximated as a linear equation. Since the electric field is proportional to voltage in TEM structure, we can bias the graphene in this range and conclude that $\mu_{\mathrm{c}}$ is proportional to bias voltage. Therefore, a cosine voltage causes a cosine chemical potential. For good performance, graphene needs to be biased in a suitable acting point that has strong nonlinearity to cause higher order terms. Before breaking point the conductivity has strong nonlinearity and after that point conductivity tends to be linear.
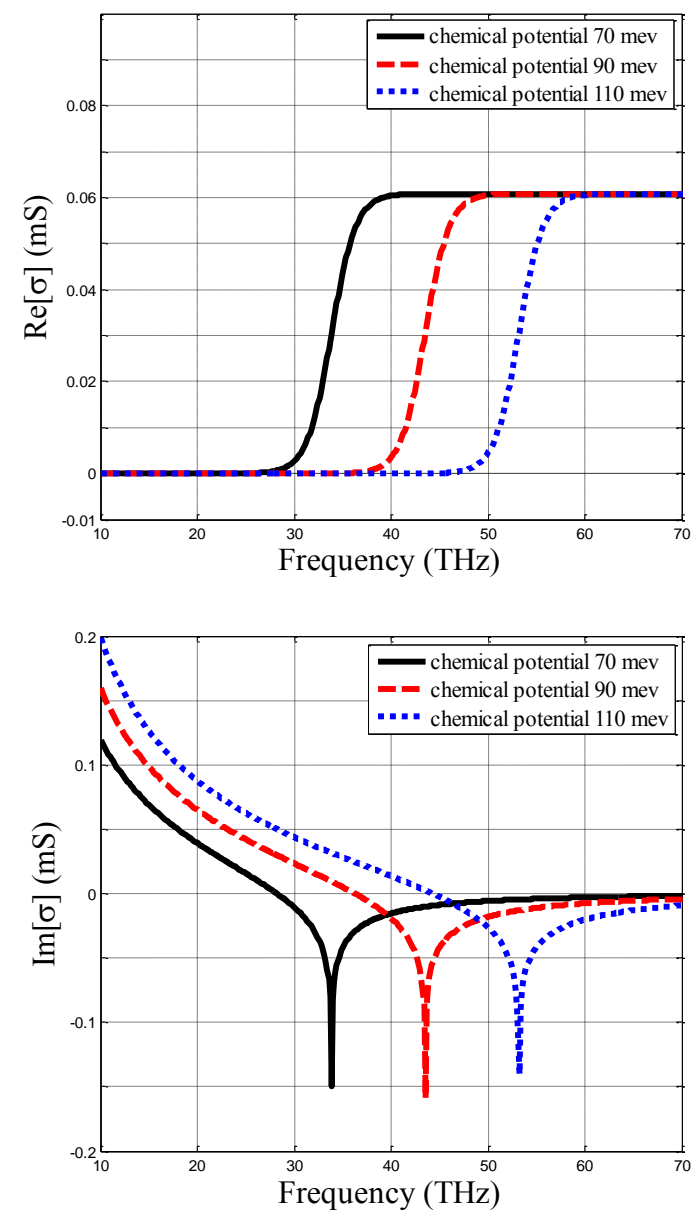

Figure 1: The real and imaginary parts of graphene conductivity versus frequency. The top picture is real part and the bottom picture represent the imaginary part for three value of chemical potential.

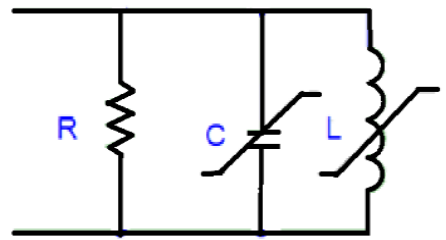

Fig 2. equivalent circuit model for the graphene where $\mathrm{R}$ represent the loss and $\mathrm{L}$ or $\mathrm{C}$ represent the resonance nature of graphene and are caused by imaginary part of its conductivity.

Our purpose is achieving equation 9 for graphene, to prove that graphene can be applicable as a mixer. Assume that a slab of graphene is biased such as figure 4 . We know that 
$j=\sigma E$

By integrating the equation 10 we have:

$$
\begin{aligned}
& \int J \cdot d s=\int \sigma E \cdot d s \\
& d s=d l \cdot d l^{\prime} \\
& \int I . d l=\int \sigma \cdot d l^{\prime} \cdot \int E \cdot d l
\end{aligned}
$$
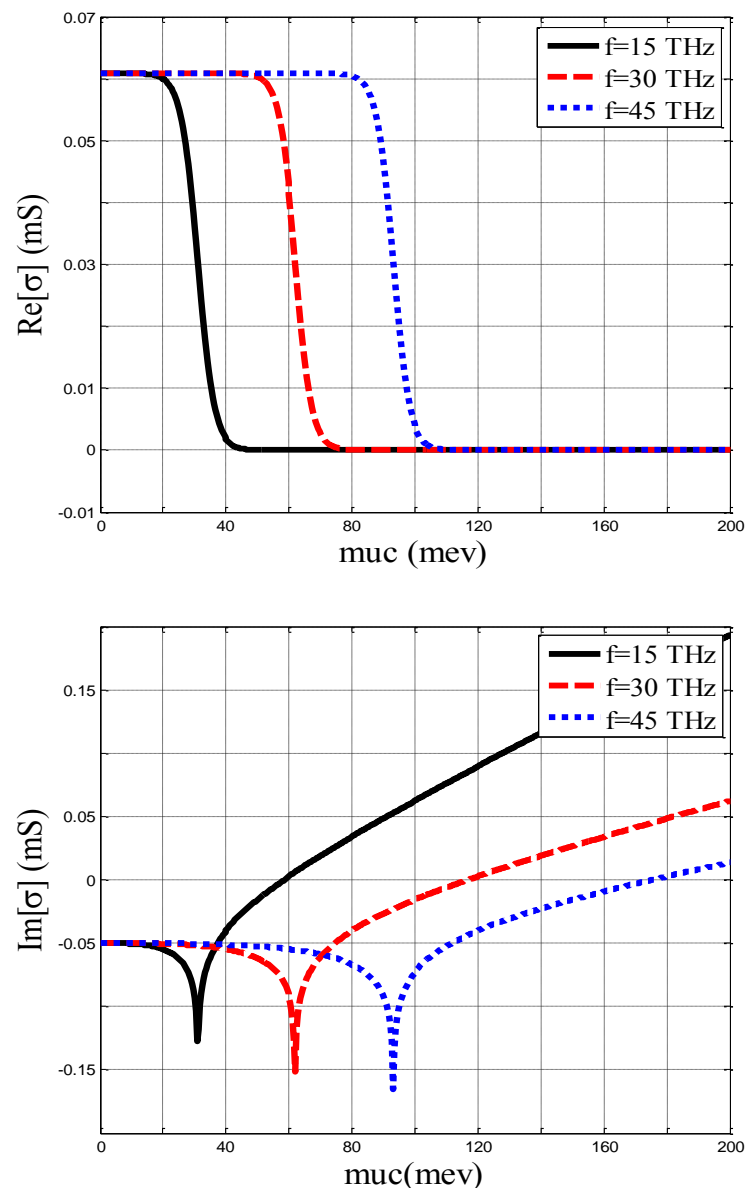

Figure 3: Optical conductivity of graphene versus voltage. The top picture shows the real part of conductivity and the bottom picture shows the imaginary part of conductivity versus chemical potential for three value of frequency.

Also we know in TEM structure:

$v=\int E \cdot d l$

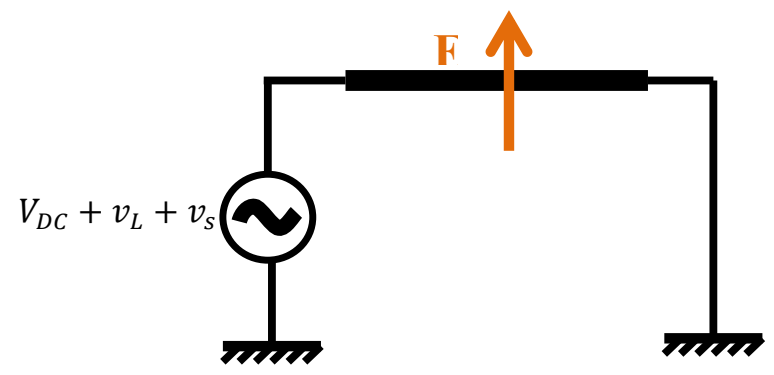

are multiplied to small signal to produce mixer components. The electric field which is shown with red arrow is bias field and should be perpendicular to surface.

By substituting equation 14 to 13 the admittance is obtained as:

$$
\begin{aligned}
& I l=\sigma l^{\prime} \cdot v \\
& I=\sigma \frac{l^{\prime}}{l} \cdot v
\end{aligned}
$$

Derivative of both sides of equation 16 will be:

$$
\frac{\partial I}{\partial v}=\frac{l^{\prime}}{l} \sigma+\frac{l^{\prime}}{l} v \frac{\partial \sigma}{\partial v}
$$

And its second derivative will be as follows:

$\frac{\partial^{2} I}{\partial v^{2}}=2 \frac{l^{\prime}}{l} \frac{\partial \sigma}{\partial v}+\frac{l^{\prime}}{l} v \frac{\partial^{2} \sigma}{\partial v^{2}}$

By substituting equation 18 in 8 different components of conductivity are expanded. At first we expand $\sigma_{\text {interband }}$.

$$
i_{1}=j \frac{l^{\prime}}{l}\left\{\begin{array}{l}
\left.\left.k \ln \frac{\frac{V_{L}}{k^{\prime}}-1}{\frac{V_{L}}{k^{\prime}}+1}\right)\right\rfloor v_{s}(t)+k\left(v_{L}(t)\right) \frac{2}{\left(\frac{V_{L}}{k^{\prime}}\right)^{2}-1} v_{s}(t)+ \\
2 k\left[\frac{2}{\left(\frac{V_{L}}{k^{\prime}}\right)^{2}-1}\right] v_{s}^{2}(t)+k\left(v_{L}(t)\right) \frac{-8 \frac{V_{L}}{k^{\prime}}}{\left(\frac{V_{L}{ }^{\prime}}{k^{\prime}}-1\right)^{2}} v_{s}^{2}(t)+\ldots
\end{array}\right\}
$$

In order to find the mixer components, we obtain the expansion of the term inside the parentheses of equation 19 as series around zero:

$$
i=j \frac{k l^{\prime}}{l}\left\{\begin{array}{l}
\left.\left(\left(\frac{V_{L}}{k^{\prime}}\right)^{2}+\left(\frac{V_{L}}{k^{\prime}}\right)^{4}+\ldots\right]-k^{\prime}\left[\left(\frac{V_{L}}{k^{\prime}}\right)+\left(\frac{V_{L}}{k^{\prime}}\right)^{3}+\left(\frac{V_{L}}{k^{\prime}}\right)^{5}+\ldots\right]\right) v_{s}(t) \\
+2\left[2-\frac{4}{2 !}\left(\frac{V_{L}}{k^{\prime}}\right)^{2}+\ldots\right] v_{s}^{2}(t)+k^{\prime}\left[1+2-\frac{4}{2 !}\left(\frac{V_{L}}{k^{\prime}}\right)^{2}+\ldots\right] \frac{v_{s}^{2}(t)}{2}
\end{array}\right\}
$$

Where

$$
\begin{aligned}
& k=\frac{e^{2}}{4 \pi \hbar} \\
& k^{\prime}=(\omega+i 2 \Gamma) \hbar \\
& V=2 \mu_{c}
\end{aligned}
$$

Also we expand the second term of the conduction, $\sigma_{\text {interband }}$ as:

$i_{2}=\frac{l^{\prime}}{l}\left\{\begin{array}{l}j A\left(\left[v_{L}+2 \ln \left(e^{-v_{L}}+1\right)\right]+v_{L}\left\lfloor 1+A\left(\frac{-2 e^{-v_{L}}}{e^{-v_{L}}+1}\right)\right]\right) v_{s}(t)+ \\ \left(2\left[1+A\left(\frac{-2 e^{-v_{L}}}{e^{-v_{L}}+1}\right)\right]+v_{L}\left[j A\left(\frac{-2 e^{-v_{L}}}{e^{-v_{L}}+1}\right)\right]\right) v_{s}^{2}(t)+\ldots\end{array}\right\}$

where,

$$
\begin{aligned}
& A=\frac{e^{2} k_{B} T}{\pi h^{2}(\omega+i 2 \Gamma)} \\
& A^{\prime}=\frac{\mu_{c}}{k_{B} T}
\end{aligned}
$$

Equation 24 is still nonlinear, so that in order to find the mixer components, we obtain the series expansion of the term inside the parentheses equation 24 as series around zero:
Figure 4: schematic of biased graphene in mixer application. The DC voltage is used for significant the suitable acting point and the large ac signal creates higher order terms that 
$i_{2}=\frac{i}{l}\left\{\begin{array}{l}j A\left(\left[v_{L}+2 \ln 2+\frac{v_{L}^{2}}{4}-\frac{1}{96} v_{L}^{4}+\ldots\right]+\left[v_{L}-2 A\left(\frac{1}{2} v_{L}+\frac{2}{2 !} v_{L}^{2}+\ldots\right)\right]\right) v_{s}(t) \\ +\left(2\left[1+A\left(\frac{1}{2} v_{L}-\frac{1}{3 ! \times 4} v_{L}^{3}+\ldots\right)-2 j A\left(\frac{1}{2} v_{L}+v_{L}^{2}+\ldots\right)\right) \frac{v_{s}^{2}(t)}{2 !}\right]\end{array}\right\}$

In equations 27 and 20 the mixer component exists, hence graphene could be used as a mixer.

To verify our conclusion the spectrum of graphene conductivity which is biased with cosine voltage as $1+\sin (\omega t)+100 * \cos (4 \omega t), \quad 1+.001 \sin (\omega t)+\cos (4 \omega t)$ and $1+10 * \sin (\omega t)+\cos (4 \omega t)$ where $\omega$ is $10 \mathrm{THz}$ is plotted in figure 5 .

As seen in figure 5 the spectrum has components in input frequencies, $\omega_{1}-\omega_{2}$ and $\omega_{1}+\omega_{2}$. Also it has unwanted components that have small amplitude in compare to desirable components. So mixer components can be easily achieved by filters.
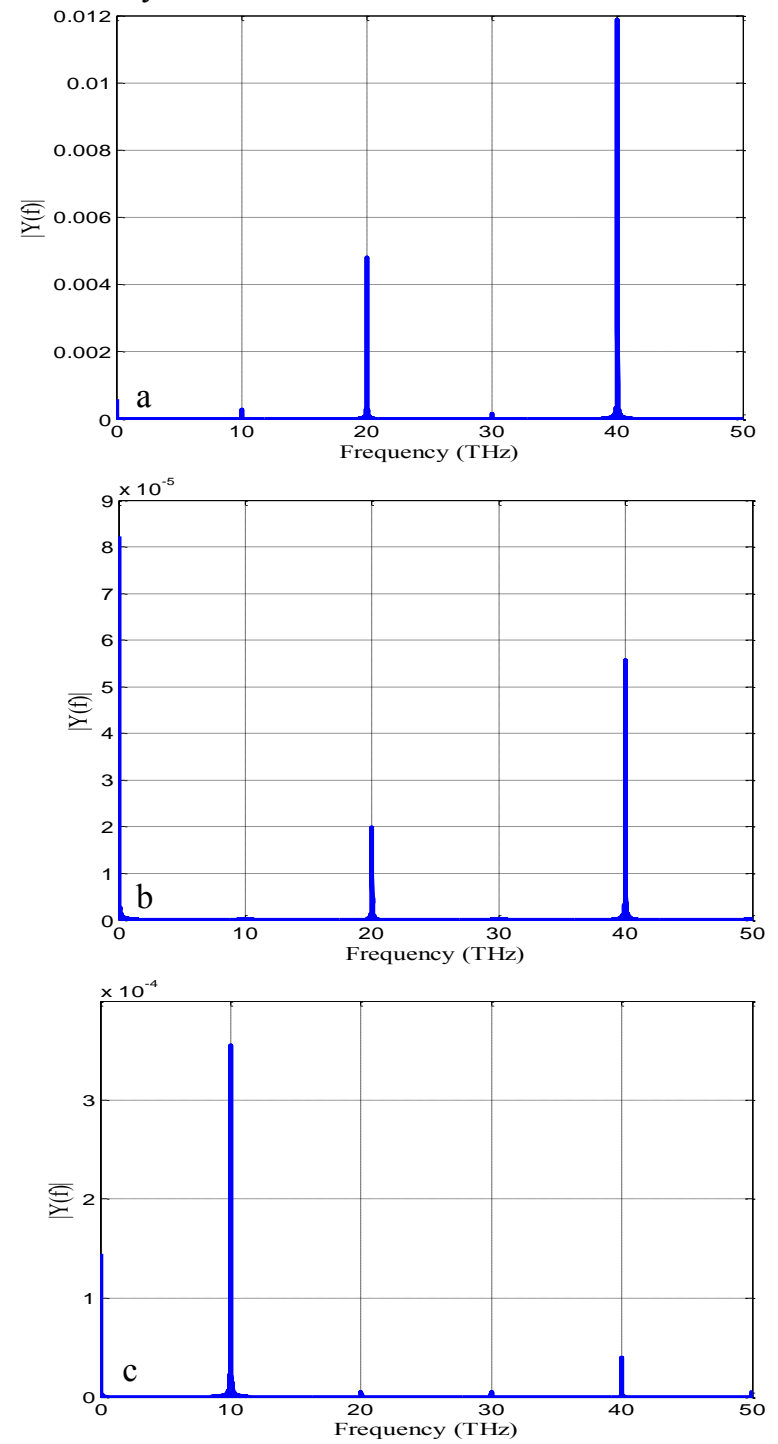

Figure 5: Spectrum of the current on the graphene when it is biased with a) $1+\sin (\omega t)+100 * \cos (4 \omega t)$ b) $1+.01 * \sin (\omega t)+$ $\cos (4 \omega t)$ and c) $1+10 * \sin (\omega t)+\cos (4 \omega t)$ where the frequency $\omega$ in the bias voltage is $10 \mathrm{THz}$, chemical potential is $125 \mathrm{mev}$, scattering rate is $0.43 \mathrm{mev}$ and temperature is $20 \mathrm{~K}$.

\section{Conclusions}

Graphene has unique physical properties that create controllable nonlinear conductivity. Using these properties we study the possibility of using graphene as a mixer in $\mathrm{THz}$ and infrared bands. To study electronic properties of graphene using quantum relations, different values of the electrical conductivity from $10 \mathrm{THz}$ to $50 \mathrm{THz}$ are obtained. In this article, by writing small signal relations around the bias voltage and extracting the electrical conductivity, it is proved that a slab of biased graphene can be used as a mixer. Because the equivalent circuit of graphene is a combination of a non-linear capacitance and a resistance (since taylor expansions have complex coefficients), we have a phase shift in the mixer component.

\section{References}

[1] Nikitas Papasimakis, Zhiqiang Luo, Ze Xiang Shen, Francesco De Angelis, Enzo Di Fabrizio, Andrey E. Nikolaenko, and Nikolay I. Zheludev," Graphene in a Photonic Metamaterial", Optics Express, 8353-8360, 2010.

[2] S. A. Mikhailov, K. Ziegler, "A New Electromagnetic Mode in Graphene”, Phys. Rev. Lett. 66, 016803, 2004.

[3] David R. Andersen, "Graphene-based long-wave infrared TM surface plasmon modulator”, JOSA , Vol. 24, Issue 4, pp. 818-823, 2010.

[4] V P Gusynin, S G Sharapov and J P Carbotte, "Magnetooptical conductivity in graphene", J. Phys.: Condens. Matter 16 026222,pp 1-26, 2004.

[5] V.P. Gusynin and S.G. Sharapov, "Transport of Dirac Quasiparticles in Graphene: Hall and Optical Conductivities", cond-mat,0512154, Phys Rev. B 43, 245411, 2006.

[6] V.P. Gusynin, S.G. Sharapov and J.P. Carbotte, "Unusual Microwave Response of Dirac Quasiparticles in Graphene", cond-mat/0603264, Phys. Rev. Lett. 66, 256802 (2006).

[7] T. Stauber, N. M. R. Peres and A. K. Geim, Optical Conductivity of Graphene in The Visible Region of The Spectrum, physical review, 085432-1-802008.

[8] G. Y. Slepyan, S. A. Maksimenko, A. Lakhtakia, O. Yevtushenko, and A. V. Gusakov, "Electrodynamics of carbon nanotubes: Dynamic conductivity, impedance boundary conditions, and surface wave propagation," Phys. Rev. B, vol. 60, pp. 14136-14146, 1666. 
[9] Jean-Noel Fucus, Mark Oliver Goerbig, "Introduction to the Physical Properties of Graphene", Lecture Notes, 2008.

[10] A V Eletskii, I M Iskandarova, A A Knizhnik, D N Krasikov , "Graphene: Fabrication Methods and Thermophysical Properties", Physics , pp 224 - 258 , 2011.

[11] Ashkan Vakil , Nader Engheta," One-Atom-Thick IR Metamaterials and Transformation Optics Using Graphene",accepted in physics.optics.

[12] Vakil, A, and N.Engheta," Transformation Optics Using Graphene", Science,Vol. 332 no. 6035, pp. 12911294 ,June 2011.

[13] Westervelt. R.M, "Graphene Nanoelectronics", science, Vol. 320 no. 5874 pp. 324-325, April 2008.

[14] Y. M. Lin, C. Dimitrakopoulos, K. A. Jenkins, D. B. Farmer, H. Y. Chiu, A. Grill, and P. Avouris, "100 GHz transistors from wafer scale epitaxial graphene," Science, vol. 327, no. 5966, p. 662, Feb. 2010.

[15] Habibpour.O, S.Cherednichenko ,J.Vukusic, K.Yhland and J.Stake "A SubharmonicGraphene FET Mixer", Electron Device Let. IEEE, pp 71-74, Jan. 2012.

[16] Hanson.G.W, "Dyadic Green's Functions for an Anisotropic Non-Local Model of iased Graphene", IEEE 2008.

[17] Andrey M. arychev "Superconductor-InsulatorSuperconductor $\mathrm{THz}$ Mixer Integrated with a Superconducting Flux-Flow Oscillator", PHD thesis, Delft university of technology, Netherland, 2005.

[18] Mass.S.A, "Analysis of Nonlinear Circuits" , Artech House, 1988. 